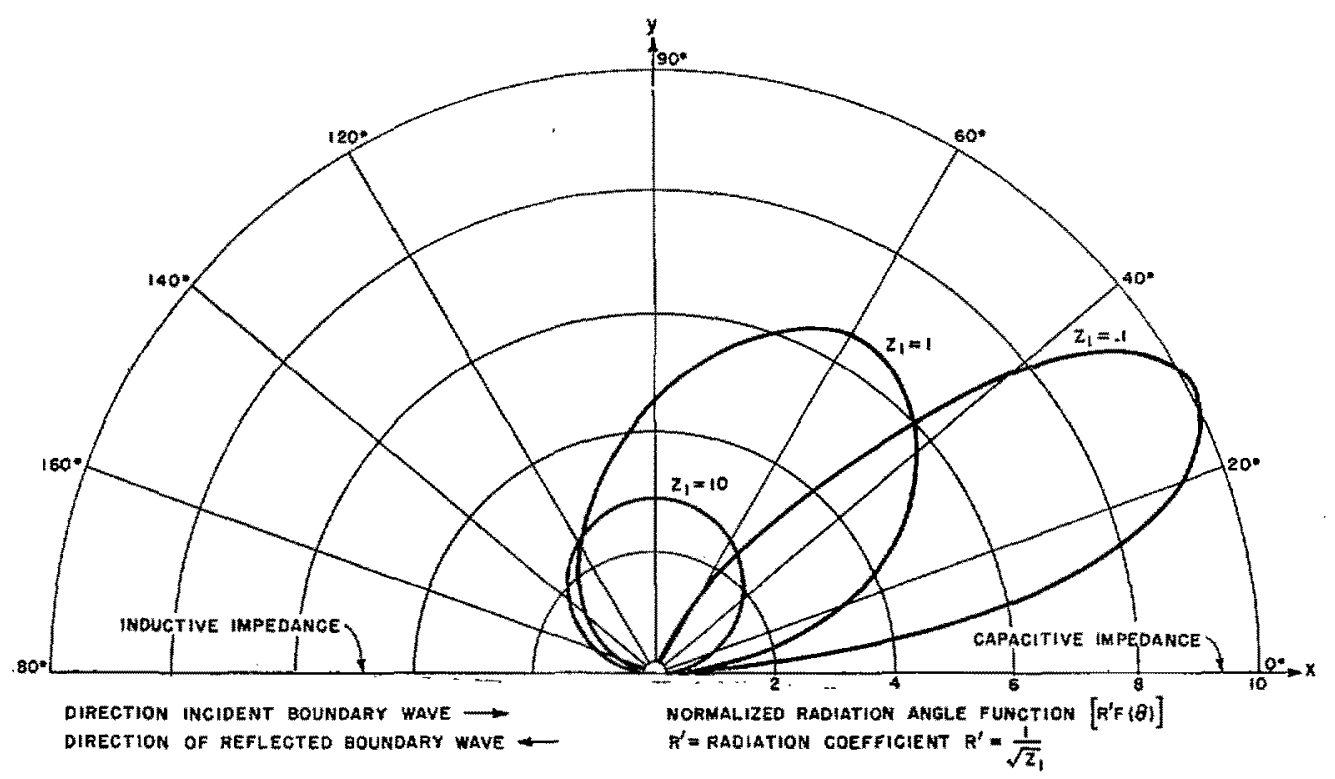

Fig. 4. The radiation pattern.

part of the impedance $(x<0)$ becomes large within a few wavelengths. This results in a boundary wave which is tightly coupled to the surface and is consequently not easily transformed into radiation.

Figure 4 shows the radiation pattern for different values of $z_{1}$. For tightly coupled boundary waves (high $z_{1}$ ) the pattern is broad. For loosely coupled boundary waves (low $z_{1}$ ) the pattern is more directive, with the maximum pointing closer and closer to the positive $x$ axis.

\section{ACKNOWLEDGMENT}

The author wishes to express his gratitude to Dr. C. Marcinkowski for his many and valuable suggestions.

\title{
Radiation Resistance of an Oscillating Dipole in a Moving Medium
}

\author{
P. DALY, K. S. H. LEE, AND C. H. PAPAS, MEMBER, IEEE
}

\begin{abstract}
The radiation resistance of an oscillating electric dipole immersed in a moving medium is calculated by Brillouin's "EMF method." Two cases are studied in detail: in case 1) the moving medium is simple, and in case 2) the moving medium is an ionized gas (plasma). It is found that in case 1), the motion of the medium tends to increase the radiation resistance, whereas in case $2)$, the motion of the medium tends to decrease the radiation resistance. A comparison is made between Frank's well-known theory of the radiation from an oscillating dipole moving through a material medium and the results obtained in this paper.
\end{abstract}

Manuscript received August 27, 1964; revised February 15, 1965. The research in this paper is supported by the U. $S$. Air Force Office of Scientific Research.

$P$. Daly is with the Dept. of Mathematics, Lniversity of Glasgow, Scotland. He was formerly with the Antenna Lab., California Institute of Technology, Pasadena, Calif.

K. S. H. Lee and C. H. Papas are with the Antenna Lab., California Institute of Technology, Pasadena, Calif.

\section{INTRODUCTION}

II ANTENNA THEORY the radiation resistance of the dipole antenna plays an important role since it is effectively the terminating resistance of the transmission line supplying the antenna. The radiation resistance of an elementary dipole radiating in vacuum has been known for some time, and more recent investigations into the subject have dealt with more complicated situations such as dipole radiation in anisotropic media [1], [2] and in dissipative media [3]. However, no attention has been given in the literature to finding the radiated power from a dipole in a medium moving uniformly with respect to the source. Recently, Lee and Papas [4], [5] have formulated the problem of electromagnetic radiation in the presence of a moving medium. 
In the present paper, we apply their theory to calculate the radiation resistance of an elementary dipole-1) in a simple (i.e., nondispersive, lossless, homogeneous, and isotropic) moving medium, and 2) in a moving ionized gas (plasma).

The total time-average power radiated by a source may be calculated by either Brillouin's "EMF method" [6] or Poynting's vector method. To formulate these two methods quantitatively we note that in an inertial frame $K$ at rest with respect to an electromagnetic source $J$ the Maxwell equations are

$$
\begin{array}{ll}
\nabla \times E=-\frac{\partial}{\partial t} B & \nabla \times H=J+\frac{\partial}{\partial t} D \\
\nabla \cdot B=0 & \nabla \cdot D=\rho .
\end{array}
$$

These equations are valid regardless of the state of the medium surrounding the source. However, they are quite meaningless until we introduce constitutive relations that connect the four field vectors $E, B, D, H$. In the present paper we assume that in the rest frame $K^{\prime}$ of the medium moving past the source with velocity $v$ the electromagnetic properties of the medium are described by the constitutive parameters $\epsilon^{\prime}$ (dielectric constant) and $\mu^{\prime}$ (permeability). This assumption, according to Mikowski's electrodynamics of moving media, leads to the following constitutive relations in $K$ :

$$
\begin{aligned}
& D+\frac{1}{c^{2}} v \times H=\epsilon^{\prime}(E+v \times B) \\
& B-\frac{1}{c^{2}} v \times E=\mu^{\prime}(H-v \times D)
\end{aligned}
$$

where $c$ is the vacuum speed of light.

For harmonic time-dependence $\left(e^{-i \omega t}\right)$, the Maxwell equations (1a) yield the following relation

$$
\begin{aligned}
\frac{1}{2} \operatorname{Re} \int_{S}\left(E \times H^{*}\right) \cdot n d S & =-\frac{1}{2} \operatorname{Re} \int_{V} E \cdot J^{*} d V \\
& -\frac{\omega}{2} \operatorname{Im} \int_{V}\left(H^{*} \cdot B-E \cdot D^{*}\right) d V
\end{aligned}
$$

where $\operatorname{Re}$ and Im denote, respectively, the real and imaginary parts, and $n$ is the unit outward normal to the surface $S$ surrounding a volume $V$ which includes the source $J$. By virtue of the constitutive relations (1b), the second term on the right-hand side of (1c) is identically zero, and we see that the total time-average power $P$ is given by the surface integral

$$
P=\frac{1}{2} \operatorname{Re} \int_{S}\left(E \times H^{*}\right) \cdot n d S
$$

or by the volume in tegral

$$
P=-\frac{1}{2} \operatorname{Re} \int_{V} E \cdot J^{*} d V
$$

Thus, we see that $P$ may be calculated by using (1d), i.e., by the Poynting vector method, or by using expression (1e), i.e., by Brillouin's ENF method. Both methods will, of course, yield the same result, but in the present instance where the source is an oscillating dipole it is easier to use Brillouin's method.

\section{Power Radiated in a Simple Moving Medium}

To compute by Brillouin's method the total timeaverage power $P$ radiated by an oscillating dipole immersed in a simple moving medium, we need to know only the electric field $E$ of the dipole. Since the vector potential $A$ and the scalar potential $\phi$ for such a dipole source are already known [4], $E$ can be obtained through the use of the relation $E=-\nabla \phi+i \omega A$. Thus we find that the electric field $E$ of an electric dipole of moment $\boldsymbol{p}$ is given by

$$
E=\omega^{2} \mu^{\prime} p \cdot L G
$$

Here the dyadic operator $L$ has the form

$$
L=u+\frac{\kappa^{\prime} \gamma^{2}}{n^{\prime 2}} v v+\frac{i \kappa^{\prime} c^{2} \gamma^{2}}{\omega n^{\prime 2}}(\nabla v+v \nabla)+\frac{1}{k^{2}} \nabla \nabla
$$

where $\kappa^{\prime}=\left(n^{\prime 2}-1\right) / c^{2}, n^{\prime}=c\left(\mu^{\prime} \epsilon^{\prime}\right)^{1 / 2}, k=n^{\prime} a \omega / c$, $a^{2}=\left(1-\beta^{2}\right) /\left(1-n^{\prime 2} \beta^{2}\right), \beta=v / c, \gamma=\left(1-\beta^{2}\right)^{-1 / 2}$, and $\boldsymbol{u}=$ unit dyadic. Also the Green's function $G$ for the case where $n^{\prime} \beta<1$ is known to be

$$
\begin{array}{r}
G=\frac{a}{4 \pi} \exp \left[-i\left(\frac{b k}{v}\right) v \cdot r\right] \\
\frac{\exp \left[i k \sqrt{\left.r^{2}+\frac{a^{2}-1}{v^{2}}(v \cdot r)^{2}\right]}\right.}{\sqrt{r^{2}+\frac{a^{2}-1}{v^{2}}(v \cdot r)^{2}}}
\end{array}
$$

where

$$
b=a \beta \gamma^{2}\left[\left(n^{\prime 2}-1\right) / n^{\prime}\right] .
$$

Substituting (2a) into (1e), we find that the total timeaverage radiated power is given by

$$
P=\frac{\omega^{3} \mu^{\prime}}{2} \operatorname{Im} \int p \cdot L G(r) \cdot p \delta(r) d V
$$

where $\delta(\boldsymbol{r})$ is the Dirac delta function. If one attempts to evaluate this integral by taking the value of the integrand at the origin, he finds that $P$ is infinite. It is pertinent to note that a similar infinity arises also in the evaluation of the radiation resistance of a dipole immersed in a magneto-ionic medium [2]. It seems that this "infinity catastrophe" is an outcome of the nonisotropic nature of the ambient medium. In the present case the infinity is removed by noting that $\delta(\boldsymbol{r})=\delta(x) \delta(y) \delta(z)$ and that $\delta(x)=\delta(-x), \delta(y)=\delta(-y)$, 
$\delta(z)=\delta(-z)$. Thus, all the odd functions of $x, y, z$ in the integrand vanish on integration. The justification of removing the singularity in this way rests on the fact that the result so obtained checks with that derived by use of the Poynting vector method (see Appendix).

We now proceed to find $P$ by evaluating (2d) for two dipole orientations. In one orientation the dipole is lined up with the moving medium, and in the other the dipole is normal to the direction of flow of the medium which is taken to be along the $z$ axis of the coordinate system.

In the parallel orientation $(p \| v)$ we have $p=p_{z} e_{z}$, and, hence, $(2 \mathrm{~d})$ for the total time-average radiated power reduces to

$$
\begin{aligned}
& P_{\mathrm{il}}=\frac{\omega^{3} p_{z}{ }^{2} \mu^{\prime}}{2} \operatorname{Im} \int {\left[1+\frac{b \beta}{a n^{\prime}}+\frac{2 i b}{k} \frac{\partial}{\partial z}+\frac{1}{k^{2}} \frac{\partial^{2}}{\partial z^{2}}\right] } \\
& \cdot G(\boldsymbol{r}) \delta(\boldsymbol{r}) d V .
\end{aligned}
$$

Carrying out the integration we obtain

$$
P_{\text {! }}=\left(\frac{1-\beta^{2}}{1-n^{\prime 2} \beta^{2}}\right)^{2} P_{0}
$$

where $P_{0}\left(=\omega^{4} p_{z}^{2} \mu^{\prime} \sqrt{\mu^{\prime} \epsilon^{\prime} / 12 \pi}\right)$ is the total time-average power the dipole would radiate if the medium were not in motion. The corresponding expression for the radiation resistance of the dipole is

$$
R_{!}=\left(\frac{1-\beta^{2}}{1-n^{\prime 2} \beta^{2}}\right)^{2} R_{0}
$$

where $R_{0}$ is the radiation resistance when the medium is not in motion.

In the perpendicular orientation $(p \perp v)$ we choose $p=p_{x} e_{x}$, and hence (2d) for the total time-average radiated power becomes

$$
P_{\perp}=\frac{\omega^{3} p_{x}^{2} \mu^{\prime}}{2} \operatorname{Im} \int\left(1+\frac{1}{k^{2}} \frac{\partial^{2}}{\partial x^{2}}\right) G(r) \delta(r) d V
$$

which in turn yields

$$
P_{\perp}=\left(\frac{1-\beta^{2}}{1-n^{\prime 2} \beta^{2}}\right) P_{0} .
$$

The radiation resistance in this case is given by

$$
R_{\perp}=\left(\frac{1-\beta^{2}}{1-n^{\prime 2} \beta^{2}}\right) R_{0}
$$

For both parallel and perpendicular orientations we see that for $n^{\prime}>1$ the radiated power and the radiation resistance exceed their stationary values. This means that to maintain the same dipole moment $p$ and angular frequency $\omega$ as in the stationary case, more power has to be fed into the dipole.

We recall that Frank [7] has considered the complementary problem of an oscillating dipole traveling uni- formly through a material medium. By using the Poynting vector method he found in the rest frame $K^{\prime}$ of the medium that the total time-average radiated power is given by

$$
P_{!^{\prime}}^{\prime}=\left(\frac{1-\beta^{2}}{1-n^{2} \beta^{2}}\right)^{3} P_{0}
$$

for parallel orientation, and by

$$
P_{\perp}^{\prime}=\left(\frac{1-\beta^{2}}{1-n^{\prime 2} \beta^{2}}\right)^{2} P_{0}
$$

for perpendicular orientation. Comparing (2k) and (21) with (2f) and (2i), we see that his results exceed ours by a factor $\left(1-\beta^{2}\right) /\left(1-n^{\prime 2} \beta^{2}\right)$. However, this discrepancy is only an apparent one and can be explained as follows. The power radiated by the dipole, as measured in the rest frame $K^{\prime}$ of the medium, is the sum of two parts. One part is the electromagnetic radiation which is equal to our expression (2f) or (2i), while the other part is due to the mechanical work done on the dipole to keep it moving uniformly through the medium. This mechanical work done appears in the form of electromagnetic radiation. In the rest frame $K$ of the dipole, however, only the first part contributes to the radiated power; the second part contributes nothing because the dipole is at rest.

The mechanical force necessary to keep the dipole stationary in $K$ is equal in magnitude but opposite in direction to the reaction force which can be calculated with the help of Minkowski's stress tensor. Then, by a proper Lorentz transformation, one can obtain the mechanical force required to keep the dipole moving uniformly in reference frame $K^{\prime}$. From a knowledge of this mechanical force in $K^{\prime}$ one can deduce the part of the mechanical work done on the dipole which appears in the form of electromagnetic radiation in $K^{\prime}$.

So far we have confined our attention to situations where $n^{\prime} \beta<1$. It is pertinent to note that when $n^{\prime} \beta \geq 1$ the radiation is of the Cerenkov type, i.e., the fields are confined within a conical region and are infinite on the conical surface [4], and the total radiated power turns out to be infinite. The infinite value arises from the assumption that the medium is nondispersive.

\section{Power Radiated in a Moving Ionized Gas}

Now we consider the case where the moving medium is an ionized gas. In the rest frame $K^{\prime}$ of the gas the constitutive parameters are $\mu^{\prime}=\mu_{0}$ and $\epsilon^{\prime}=\epsilon_{0}\left(1-\omega_{p}{ }^{2} / \omega^{\prime 2}\right)$ where $\epsilon_{0}, \mu_{0}$ are the free-space dielectric constant and permeability, $\omega_{p}^{\prime}$ is the plasma frequency, and $\omega^{\prime}$ is the frequency of the radiation.

We recall that to compute by Brillouin's method the total time-average power radiated by the dipole we need to know the electric field $E$ of the dipole. It has been 
found that, in the rest frame $K$ of the dipole, $E$ is related to the dipole moment $p$ through the relation [5]

$$
\begin{aligned}
E= & \mu_{0} c^{2} \boldsymbol{p} \cdot\left(k_{0}^{2} u+\nabla \nabla\right) G^{(0)} \\
& -i \omega \mu_{0} c \beta\left(e_{z} p \cdot \nabla+e_{z} \cdot p \nabla\right) G^{(1)} \\
& -\mu_{0} c^{2} p \cdot\left(k_{0}^{2} \beta^{2} e_{z} e_{z}-\nabla \nabla\right) G^{(1)}
\end{aligned}
$$

where $u=$ unit dyadic, $k_{0}=\omega / c$, and $\omega=$ angular frequency of dipole. The functions $G^{(0)}, G^{(1)}$ are defined by

$$
\begin{aligned}
G^{(0)} & =\frac{e^{i k r}}{4 \pi r} \\
G^{(1)} & =\frac{i k_{p}{ }^{2}}{8 \pi} \gamma^{2} \int_{-\infty}^{\infty} \frac{B_{0}(1)\left(\rho \sqrt{\left.k^{2}-s^{2}\right)}\right.}{\gamma^{2}\left(k_{0}-\beta s\right)^{2}-k_{p}{ }^{2}} e^{i s z} d s
\end{aligned}
$$

where $k_{p}=\omega_{p} / c, \omega_{p}=$ plasma frequency, $k^{2}=k_{0}{ }^{2}-k_{p}{ }^{2}$, and $\rho^{2}=x^{2}+y^{2}$.

To compute the total time-average power radiated by the dipole, we substitute (3a) into (1e) and obtain for $p \| v$ the following expression

$$
\begin{gathered}
P_{\|}=\frac{\omega^{3} \mu_{0} p_{z}^{2}}{2} \operatorname{Im} \int\left(1+\frac{1}{k_{0}^{2}} \frac{\partial^{2}}{\partial z^{2}}\right) G^{(0)} \delta(r) d V \\
-\omega^{2} \mu_{0} p_{z}^{2} c \beta \operatorname{Re} \int \frac{\partial}{\partial z} G^{(1)} \delta(r) d V \\
+\frac{\omega \mu_{0} p_{z}^{2}}{2} c^{2} \operatorname{Im} \int \frac{\partial^{2}}{\partial z^{2}}-\beta^{2} k_{0}^{2} G^{(1)} \delta(\boldsymbol{r}) d V .
\end{gathered}
$$

The first term $P_{\|}^{(1)}$ on the right.-hand side of (3d) is easily evaluated and is given by

$$
P_{\|}^{(1)}=\frac{\omega^{3} \mu_{0} p_{2}{ }^{2} k}{8 \pi}\left(1-\frac{k^{2}}{3 k_{0}^{2}}\right) .
$$

To evaluate the second term $P_{1}^{(2)}$ on the right-hand side of (3d), we note that for $s>|k|$ the integral

$$
\int \frac{\partial}{\partial z} G^{(1)} \delta(r) d V
$$

has no real part since the Hankel function $H_{0}^{(1)}$ arising in the expression for $G^{(1)}$ is purely imaginary for $s>|k|$. Thus $P_{\|^{(2)}}$ reduces to

$$
\begin{aligned}
P_{U}(2)= & \frac{\omega^{2} \mu_{0} \phi z_{z}{ }^{2} c \beta \gamma^{2} k_{p}{ }^{2}}{8 \pi} \operatorname{Re} \int \mathrm{C} . \mathrm{P} . \int_{-k}^{k} \frac{s H_{0}{ }^{(1)}\left(\rho \sqrt{\left.k^{2}-s^{2}\right)}\right.}{\gamma^{2}\left(k_{0}-\beta s\right)^{2}-k_{p}^{2}} \\
& \cdot e^{i s z \delta(\boldsymbol{x}) d s d V,}
\end{aligned}
$$

where C.P. denotes the Cauchy principle value. We now write $H_{0}^{(1)}=J_{0}+i Y_{0}$ and interchange the order of integration. Then it can be seen that the value of the integral (3f) corresponding to $Y_{0}$ has no real part, and hence $P_{\|}^{(2)}$ becomes

$P_{\|}^{(2)}=\frac{\omega^{2} \mu_{0} \hat{p}_{z}^{2} c \beta \gamma^{2} k_{p}{ }^{2}}{8 \pi}$ C.P. $\int_{-k}^{k} \frac{s d s}{\gamma^{2}\left(k_{0}-\beta s\right)^{2}-k_{p}^{2}} \cdot$
Similarly the third term $P_{\|^{(3)}}$ on the right-hand side of (3d) yields

$P_{\|}{ }^{(3)}=-\frac{\omega^{3} \mu_{0} p_{z}^{2} \gamma^{2} k_{p}{ }^{2}}{16 \pi k_{0}{ }^{2}}$ C.P. $\int_{-k}^{k} \frac{s^{2}+k_{0}^{2} \beta^{2}}{\gamma^{2}\left(k_{0}-\beta s\right)^{2}-k_{p}^{2}} d s$.

After performing the integrations in (3g) and (3h) we find that the total time-average power radiated by the dipole is given by

$$
P_{\mathbb{I}}=\frac{3}{2} P_{0}\left[1-\frac{k^{2}}{3 k_{0}{ }^{2}}-\frac{k_{p}{ }^{2}}{2 k_{0}{ }^{2} \beta^{2}}(2+f)\right]
$$

where $f$ is defined by

$$
\begin{aligned}
f=\frac{1}{k k_{p} \beta \gamma^{3}}\left[2 \gamma k_{0} k_{p}\right. & \log \frac{k-\beta k_{0}}{k+\beta k_{0}} \\
& \left.-\left(k_{0}^{2}+\gamma^{2} k_{p}^{2}\right) \log \frac{k-\beta \gamma k_{p}}{k+\beta \gamma k_{p}}\right] .
\end{aligned}
$$

For the case $p \perp v$, we choose $p=p_{x} e_{x}$ and find in a similar manner that the total time-average radiated power $P_{\perp}$ is given by

$$
P_{\perp}=\frac{3}{2} P_{0}\left[1-\frac{k^{2}}{3 k_{0}^{2}}+\frac{k_{3}^{2}}{4 k_{0}^{2} \beta^{2}}\left(2+\gamma^{2} f\right)\right]
$$

where $f$ is again defined by $(3 \mathrm{j})$.

Expressions (3i) and (3k) can be cast into power series in $\beta$. Keeping terms up to $\beta^{2}$ we obtain

$$
\begin{aligned}
& P_{\|}=P_{0}\left[1-\frac{k_{p}{ }^{2}\left(k^{2}+k_{0}{ }^{2}\right)}{5 k^{2} k_{0}{ }^{2}} \beta^{2}+O\left(\beta^{4}\right)\right] \\
& P_{\perp}=P_{0}\left[1-\frac{k_{p}^{2}\left(3 k_{0}{ }^{2}-4 k_{p}^{2}\right)}{10 k^{2} k_{0}^{2}} \beta^{2}+O\left(\beta^{4}\right)\right] .
\end{aligned}
$$

Examination of (3i), (3k), and (3j) shows that $P_{9}$ and $P_{\perp}$ are invariant under the transformation $\beta \rightarrow-\beta$. This means that $P_{1}$ and $P_{\perp}$ have power-series expansions which involve only even powers of $\beta$. For small $\beta$ we see from (31) and $(3 \mathrm{~m})$ that in the parallel case the radiated power is always smaller than its stationary value, and in the perpendicular case this is still true provided that $\omega / \omega_{p}>2 / \sqrt{3}$. The reason may be that, in view of (2f) and (2i), the refractive index of a completely ionized gas, as measured in the rest frame of the gas, is less than unity. But this is by no means the whole story, for an ionized gas is a dispersive medium and, when it is in motion, its interaction with electromagnetic waves is by far more complicated than the interaction of electromagnetic waves with a moving nondispersive medium.

\section{APPENDIX}

In this appendix we wish to show that the radiated power from an oscillating dipole in a moving simple 
medium can also be obtained by the Poynting vector method. The expression for the total time-average radiated power from an elementary electric dipole is

$$
P=\frac{1}{2} \operatorname{Re} \int_{S}\left(E \times H^{*}\right) \cdot n d S .
$$

To evaluate this integral we need to know the far-zone expressions for the fields $E$ and $H$. First we derive the far-zone expressions for $E$ and $B$ from the vector and scalar potentials $A$ and $\phi$ given in Lee and Papas [4]. Then we find the far-zone $H$ by means of (1b).

In the case $p \| v$, we find that the far-zone spherical components of $E$ and $H$ are given by

$$
\begin{aligned}
& E_{\theta}=\omega^{2} \mu^{\prime} p_{z} a^{2} \sin \theta\left(\sin ^{2} \theta+a^{2} \cos ^{2} \theta\right)^{-1} G \\
& E_{r}=E_{\phi}=0 \\
& H_{\phi}=-\omega p_{z} k \sin \theta\left(\sin ^{2} \theta+a^{2} \cos ^{2} \theta\right)^{-1 / 2} G \\
& H_{r}=H_{\theta}=0 .
\end{aligned}
$$

Substituting expressions (5) into (4) we obtain

$$
P_{11}=a^{5} P_{0} \frac{3}{4} \int_{0}^{\pi} \frac{\sin ^{3} \theta d \theta}{\left(\sin ^{2} \theta+a^{2} \cos ^{2} \theta\right)^{5 / 2}} .
$$

After performing the integration we arrive at (2f).

In the case $p \perp v$ we find the following expressions for the far-zone fields:

$$
E_{\theta}=\omega^{2} \mu^{\prime} p_{x} a^{2} \cos \phi \cos \theta\left(\sin ^{2} \theta+a^{2} \cos \theta\right)^{-1} G
$$

$$
\begin{aligned}
& E_{\phi}=-\omega^{2} \mu^{\prime} p_{x} \sin \phi G \\
& E_{r}=0 \\
& H_{\theta}=\omega p_{x} k \sin \phi\left(\sin ^{2} \theta+a^{2} \cos ^{2} \theta\right)^{-1 / 2} G \\
& H_{\phi}=\omega p_{x} k \cos \phi \cos \theta\left(\sin ^{2} \theta+a^{2} \cos ^{2} \theta\right)^{-1 / 2} G \\
& H_{\tau}=0 .
\end{aligned}
$$

Substituting expressions (7) into (4) we obtain

$$
\begin{aligned}
P_{1}= & a^{3} P_{0} \frac{3}{8 \pi} \int_{0}^{2 \pi} \int_{0}^{\pi} \frac{a^{2} \cos ^{2} \theta+\sin ^{2} \phi \sin ^{2} \theta}{\left(\sin ^{2} \theta+a^{2} \cos ^{2} \theta\right)^{5 / 2}} \\
& \cdot \sin \theta d \theta d \phi .
\end{aligned}
$$

After performing this integration we arrive at (2i).

\section{REFERENCES}

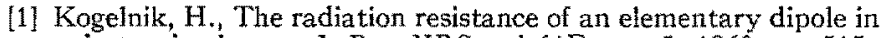
anisotropic plasma, $J$. Res. NBS, vol $64 \mathrm{D}$, no. 5,1960 , pp 515 523.

[2] Staras, H., The impedance of an electric dipole in a magnetoionic medium. IEEE Trans. on Antentas and Propagation, vol AP-12, Nov 1964, pp 695-702.

[3] King, R. W. P. and C. W. Harrison, Jr., Half-wave cylindrical antenna in a dissipative medium: current and impedance, $f$, Res. NBS, vol 64D, 1960 , pp. 365-380.

[4] Lee, K. S. H, and C. H. Papas, Electromagnetic radiation in the presence of simple moving medium, J. Math. Phys., vol 5, Dec 1964, pp 1668-1672.

[5] Antenna radiation in a moving dispersive medium, IEEE Trans. on Antennas and Propagation, vol. AP-13, September 1965.

[6] Brillouin, L., Origin of radiation resistance, Radioelectricite, Apr 1922, pp $14 \%-152$

[7] Frank, 1., Doppler effect in a refractive medium, J. Phys. USSR, vol 2 , pt 7,1943 , pp 49-67.

\title{
Plasma Simulation with an Artificial Dielectric in a Horn Geometry
}

\author{
K. E. GOLDEN, MEMBER, IEEE
}

\begin{abstract}
Abtsract-The simulation of a plasma sheath using an artificial dielectric is studied in this investigation and is applied to an antenna geometry which is similar to some configurations encountered in aerospace applications. The antenna configuration is equivalent to a horn in an infinite ground plane with an unbounded plasma layer in front of the horn. The plasma layer is simulated by a rodded medium, and the radiation patterns of the antenna system are studied experimentally at 9,10 , and $11 \mathrm{Gc} / \mathrm{s}$.

Two constructions of rodded media are utilized in the pattern measurements. The experimentally determined radiation patterns have a secondary maximum associated with the properties of the plasma sheath. More energy is radiated in the OFF-axis region when the plasma sheath is present.
\end{abstract} 1965.

Manuscript received September 21, 1964; revised February 25, The author is with the Aerospace Corp., Los Angeles, Calif.

\section{INTRODUCTION}

TMHE PURPOSE of this investigation was to study plasma simulation using a rodded medium and to study the radiation patterns of an $X$-band horn antenna in the presence of a simulated plasma sheath. The plasma sheath is assumed to be under-dense or operating above the plasma frequency. The antenna geometry is shown in Fig. 1. Several authors [1]-[4] have studied this geometry or very similar geometries previously, but to obtain a clean experiment using a laboratory plasma is very difficult. Typically, the experimental and theoretical geometries are not the same. Usually, the theoretical models are highly idealized and only approximate the actual experimental geometry be- 